\title{
Thermodynamics of Bose-Condensed Atomic Hydrogen
}

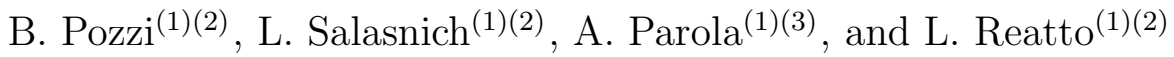 \\ (1) Istituto Nazionale per la Fisica della Materia, Unità di Milano, \\ Via Celoria 16, 20133 Milano, Italy \\ (2) Dipartimento di Fisica, Università di Milano, \\ Via Celoria 16, 20133 Milano, Italy \\ (3) Dipartimento di Scienze Fisiche, Università dell'Insubria, \\ Via Lucini 3, 23100 Como, Italy
}

\begin{abstract}
We study the thermodynamics of the Bose-condensed atomic hydrogen confined in the Ioffe-Pritchard potential. Such a trapping potential, that models the magnetic trap used in recent experiments with hydrogen, is anharmonic and strongly anisotropic. We calculate the ground-state properties, the condensed and non-condensed fraction and the Bose-Einstein transition temperature. The thermodynamics of the system is strongly affected by the anharmonicity of this external trap. Finally, we consider the possibility to detect Josephson-like currents by creating a double-well barrier with a laser beam.
\end{abstract}

PACS numbers: 03.75.Fi, 05.30.Jp, 32.80.Pj

Typeset using REVTEX 
Few years ago, Bose-Einstein condensation (BEC) has been experimentally observed in clouds of trapped alkali-metal atoms [1]. Recently, BEC has been also achieved with atomic hydrogen confined in a Ioffe-Pritchard trap [2]. That is an important result because hydrogen properties, like interatomic potentials and spin relaxation rates, are well understood theoretically. As stressed by Killian et al. [2], the s-wave scattering length of the hydrogen is very low and, compared with other atomic species, the condensate density is high, even for small condensate fractions. Moreover, due to hydrogen's small mass, the BEC transition occurs at higher temperatures than in those of alkali atoms.

In this paper we calculate the thermodynamical properties of the trapped hydrogen gas by using the quasi-classical Hartree-Fock approximation [3]. This approach is justified by the very large number of atoms (about $10^{10}$ ) in the trap and by the relatively high temperatures involved (order of $\mu \mathrm{K}$ ). Due to the anharmonic external trap, the analytical results for BEC thermodynamics obtained by Stringari et. al [3] cannot be used for quantitative predictions. Our detailed theoretical study of the hydrogen thermodynamics can give useful informations for future experiments with a better optical resolution. In the last part of the paper we discuss the criteria for macroscopic quantum tunneling and macroscopic quantum self-trapping by using a laser beam to create a double-well potential.

In the experiment reported in Ref. [2], the axially symmetric magnetic trap is modelled by the Ioffe-Pritchard potential

$$
U(\rho, z)=\sqrt{(\alpha \rho)^{2}+\left(\beta z^{2}+\gamma\right)^{2}}-\gamma,
$$

where $\rho$ and $z$ are cylindrical coordinates and the parameters $\alpha, \beta$ and $\gamma$ can be calculated from the magnetic coil geometry. In particular, for small displacements, the radial oscillation frequency is $\omega_{\rho}=\alpha / \sqrt{m \gamma}=2 \pi \times 3.90 \mathrm{kHz}$, the axial frequency is $\omega_{z}=\sqrt{2 \beta / m}=2 \pi \times 10.2$ $\mathrm{Hz}$ and $\gamma / k_{B}=35 \mu \mathrm{K}[2]$.

A dilute gas of $N$ identical hydrogen atoms is theoretically described by a bosonic field operator $\hat{\psi}(\mathbf{r}, t)$. Following a standard treatment, one can separate out the condensate part with the Bogoliubov prescription $\hat{\psi}(\mathbf{r}, t)=\Phi(\mathbf{r})+\hat{\phi}(\mathbf{r}, t)$, where $\Phi(\mathbf{r})=\langle\hat{\psi}(\mathbf{r}, t)\rangle$ is the 
macroscopic wavefunction (order parameter) of the condensate, normalized to the number $N_{0}$ of condensed atoms, and $\langle\ldots\rangle$ is the mean value in the grand-canonical ensemble. Then, in the mean-field approximation, the order parameter $\Phi(\mathbf{r})$ satisfies the following finitetemperature Gross-Pitaevskii equation $[4,5]$

$$
\left[-\frac{\hbar^{2}}{2 m} \nabla^{2}+U(\mathbf{r})+g n_{0}(\mathbf{r})+2 g n_{T}(\mathbf{r})\right] \Phi(\mathbf{r})=\mu \Phi(\mathbf{r})
$$

where $m$ is the mass of the atomic hydrogen, $\mu$ the chemical potential, and $g=4 \pi \hbar^{2} a / m$ is the scattering amplitude with $a$ the s-wave scattering length $(a=0.0648 \mathrm{~nm})$. The condensate density is $n_{0}(\mathbf{r})=|\Phi(\mathbf{r})|^{2}$ and $n_{T}(\mathbf{r})=<\hat{\phi}^{+}(\mathbf{r}, t) \hat{\phi}(\mathbf{r}, t)>$ is the thermal density of non-condensed particles, normalized to $N_{T}=N-N_{0}$. Notice that we ignore the $T=0$ quantum depletion. In fact, the corrections to the mean-field results are fixed by the gas parameter $n(\mathbf{0}) a^{3}$, where the total density $n(\mathbf{r})$ is evaluated at the center of the trap [6]. We shall show that this parameter is very small also when there are billions of atoms in the trap.

The thermal density can be calculated through the quasi-classical Hartree-Fock approximation

$$
n_{T}(\mathbf{r})=\frac{1}{\lambda^{3}} g_{3 / 2}\left(e^{-(U(\mathbf{r})+2 g n(\mathbf{r})-\mu) / k_{B} T}\right),
$$

where $g_{\alpha}(z)=\sum_{k=1}^{\infty} z^{k} / k^{\alpha}$ and $\lambda=\left(2 \pi \hbar^{2} / m k_{B} T\right)^{1 / 2}$ is the thermal length. Thus, the thermal particles behave as "non-interacting" bosons moving in the self-consistent effective potential $U(\mathbf{r})+2 g n(\mathbf{r})$, where the term $2 g n(\mathbf{r})$ is the mean-field generated by interactions with other atoms [3]. Note that the quasi-classical approximation is accurate for the experimental hydrogen cloud, which has $N=10^{10}$ atoms. Also the Hartree-Fock approximation is valid in this context because $k_{B} T$ is much larger than the chemical potential $\mu$.

In many papers, the thermodynamics of Bose-condensed dilute gases has been studied by solving the Eq. (2) and (3) with a self-consistent iterative procedure (see [6] and references therein). We shall adopt this method that, in our case, describes correctly both the highand low-temperature regimes due to the negligible effect of collective excitations in the 
thermal density. It is important to observe that mean-field predictions for trapped alkalimetal atoms are in good agreement with recent path-integral Monte Carlo calculations [7]. Moreover, in the experiments with atomic hydrogen, the gas is dilute $\left(n a^{3} \ll 1\right)$ but also strongly interacting because $N a / a_{H} \gg 1$, where $a_{H}=\left(\hbar / m \omega_{H}\right)^{1 / 2}$ with $\omega_{H}=\left(\omega_{\rho}^{2} \omega_{z}\right)^{1 / 3}$. It follows that the kinetic term of the Eq. (2) can be safely neglected, as we have verified by numerically solving Eq. (2) at zero temperature, and one gets the Thomas-Fermi condensate density

$$
n_{0}(\mathbf{r}, t)=\frac{1}{g}\left[\mu-U(\mathbf{r})-2 g n_{T}(\mathbf{r})\right],
$$

in the region where $\mu>U(\mathbf{r})+2 g n_{T}(\mathbf{r})$, and $n_{0}(\mathbf{r})=0$ outside. In practice, we study the BEC thermodynamics by solving self-consistently the Eq. (3) and (4).

First, let us consider a noninteracting gas $(g=0)$. In such a case, the formula (3) of thermal density simplifies and one can directly obtain the BEC transition temperature $T_{c}^{0}$ as a function of the total number $N$ of atoms by numerically solving the equation

$$
N=\int \frac{d^{3} \mathbf{r}}{\left(2 \pi \hbar^{2} / m k_{B} T_{c}^{0}\right)^{3 / 2}} g_{3 / 2}\left(e^{-U(\mathbf{r}) / k_{B} T_{c}^{0}}\right) .
$$

In Fig. 1 we compare the BEC transition temperature $T_{c}^{0}$ of the Ioffe-Pritchard potential with the analytic formula $T_{c}^{0, H}=0.94 \hbar \omega_{H} N^{1 / 3} / k_{B}$, that is exact in the thermodynamic limit for the harmonic potential $U=(m / 2)\left(\omega_{\rho}^{2} \rho^{2}+\omega_{z}^{2} z^{2}\right)$. Fig. 1 shows that, for a large number of atoms, $T_{c}^{0, H}$ exceeds $T_{c}^{0}$. In particular, for $N=2 \cdot 10^{10}$ the relative difference is more than $41 \%$. In the Ioffe-Pritchard trap the BEC transition temperature is strongly affected by the fact that along the cylindrical radius the quadratic behavior becomes almost linear at large distances (for a discussion of potentials with exact power laws see [8]). It means that the density of states is higher than in the harmonic case and consequently $T_{c}^{0}$ is suppressed. The critical temperature in this Ioffe-Pritchard trap can be well represented by the law $T_{c}^{0}=b N^{1 / \eta}$, where $b=5.47 \cdot 10^{-2} \mu \mathrm{K}$ and $\eta=3.51$.

The role of the interatomic interaction on the transition temperature $T_{c}$ is very small. In Fig. 1 one observes that the repulsive interaction reduces $T_{c}$ both in harmonic and IoffePritchard traps. The shift of the transition temperature $\delta T_{c}$ is in agreement with the law 
$\delta T_{c} / T_{c}=-1.3\left(a / a_{H}\right) N^{1 / 6}$, predicted by Stringari et al. [3] for the harmonic trap. These corrections are of the order of $0.1 \mu \mathrm{K}$.

In Fig. 2 we show the condensate density $n_{0}(\mathbf{r})$ and the thermal density $n_{T}(\mathbf{r})$ at $T=45$ and $47 \mu \mathrm{K}$ for $N=2.2 \cdot 10^{10}$ atoms. The thermal density shows a depletion near the origin, that is due to the presence of the condensate fraction in that region. Note that the thermal cloud fills a very large spatial region compared to the condensate one.

In Fig. 3 we present various properties of the condensate as a function of temperature for $N=2.2 \cdot 10^{10}$ atoms. We consider the number of atoms and temperatures achieved in the MIT experiment [2]. The central density and the size of the condensate are particularly interesting because can be easily detected experimentally. Our results are compatible with the experimental data: at $T=45 \pm 5 \mu \mathrm{K}$ with $N=2.2 \cdot 10^{10}$ the estimated condensate fraction is $5 \%$, the peak condensate density $4.8 \pm 1.1 \cdot 10^{15} \mathrm{~cm}^{-3}$, the condensate diameter is $d=15 \mu \mathrm{m}$ and its length $l=5 \mathrm{~mm}[2]$. We note that, as previously anticipated, the gas parameter $n(\mathbf{0}) a^{3}$ is always less than $10^{-6}$.

The condensed fraction, the energy per particle of the condensate and the chemical potential are shown in Table 1. We calculate also the two-body decay rate $\Gamma=c \int d^{3} \mathbf{r} n_{0}^{2}(\mathbf{r})$, where $c=1.1 \cdot 10^{-15} \mathrm{~cm}^{3} / \mathrm{s}[3]$. The decay time can be estimated as $\tau_{1 / 2}=7 /\left(2 \mathrm{cn}_{0}(\mathbf{0})\right)$ [3]. The experimentally measured life-time (about 5 seconds with $N_{0} / N=0.05$ ) is larger than $\tau_{1 / 2}$. As stressed in [2], the thermal gas continually replenishes the condensate as atoms are lost through two-body collisions. Thus the apparent life-time of the condensate is increased by this replenishment.

For the sake of completeness, in Fig. 4 we plot the condensate fraction $N_{0} / N$ as a function of temperature for $N=10^{8}, 10^{9}$ and $10^{10}$ atoms. Note that Hijmans, Kagan, Shlyapnikov and Walraven [9] have shown that, due to the balance between the thermalization rate and the two-body spin-relaxation decay rate, the maximum achievable equilibrium condensate fraction for hydrogen cannot be very large. Moreover, a recent theoretical paper [10] suggests the possibility of interesting nonequilibrium effects like the short-time formation of 
quasicondensate droplets. However, we belive that the equilibrium results we have shown may provide a useful guide for future experiments with a better optical resolution.

A very interesting issue is the possibility to detect macroscopic quantum tunneling (MQT) and Josephson-like oscillations of the Bose condensate in double-well traps $[11,12]$. Recently, we have shown that with ${ }^{23} \mathrm{Na}$ atoms in harmonic trap one sees only the macroscopic quantum self-trapping (MQST) of the condensate. To get outside the MQST regime it is necessary to strongly reduce the scattering length [12]. Due to its very low scattering length, atomic hydrogen is a good candidate for MQT in double-well traps.

It is easy to create a double-well trap for a cigar shaped condensate by using a laser beam [13]. The effect of a laser beam on atoms can be modelled by the following potential

$$
U_{L}(z)=U_{0} \exp \left(\frac{-z^{2}}{2 \sigma^{2}}\right)
$$

where the potential barrier $U_{0}$ is proportional to the total power of the laser beam perpendicular to the long axis of the condensate, and $\sigma$ is the beam radius [13].

Smerzi et al. [11] have shown that the time-dependent behavior of the condensate in a double-well potential depends on the parameter $\Lambda=4 E_{\text {int }} /\left|\Delta E^{0}\right|: E_{\text {int }}$ is the interaction energy of the condensate and $\Delta E^{0}$ is the kinetic+potential energy splitting between the ground state and the quasi-degenerate odd first excited state of the GP equation [12,13]. Let $z=\left(N_{1}-N_{2}\right) / N$ be the fractional population imbalance of the condensate in the two wells. For a fixed $\Lambda(\Lambda>2)$ there exists a critical $z_{c}=2 \sqrt{\Lambda-1} / \Lambda$ such that for $0<z<z_{c}$ there are Josephson-like oscillations of the condensate. For $z \ll z_{c}$ these oscillations are harmonic with period $\tau=\tau_{0} / \sqrt{1+\Lambda}$, where $\tau_{0}=2 \pi \hbar /\left|\Delta E^{0}\right|$. Instead for $z_{c}<z \leq 1$ there is MQST of the condensate: even if the populations of the two wells are initially set in an asymmetric state $(z \neq 0)$ they maintain the original population imbalance without transferring particles through the barrier $[11,12]$.

By solving the zero-temperature GP equation (2) with the steepest-descent method in the double-well trap given by Eq. (1) and Eq. (6), we calculate the parameter $\Lambda$ for the condensate of hydrogen atoms in the tunneling region. In Table 2 it is shown that, for a 
small number of condensed atoms (about $10^{4}$ ), the MQT is possible with a relatively large fractional population imbalance $z$. For this number of condensed atoms, the period $\tau$ of oscillation is smaller than the life-time $\tau_{1 / 2}$ of the condensate, that is about 50 seconds. For a larger number of atoms, the parameter $\Lambda$ quickly grows and the condensate remains self-trapped. Note that at nonzero temperature, BEC depletion and thermal fluctuations will slightly modify the parameters of the tunneling and will damp the coherent oscillations. Nevertheless, as shown by Zapata, Sols and Leggett [14], the effect of damping is negligible for temperatures lower than about $10 \hbar \omega_{H}$.

In this paper we have studied the thermodynamics of the atomic hydrogen in a IoffePritchard trap. We have found that the BEC transition temperature is strongly dependent on the anharmonicity of the trap while the effect of repulsive interaction is small. We have calculated the properties of the Bose condensate at various temperatures. Our results are consistent with available experimental data, which still have a large error. The present paper should be very useful for future experiments because it gives detailed information on the Bose condensate at temperatures not achieved so far. Finally, we have considered the inclusion of a laser beam to produce Josephson-like currents in the resulting double-well trap. Our calculations suggest that with a small number of condensed atoms (about $10^{4}$ ) the macroscopic quantum tunneling can be observed.

\section{ACKNOWLEDGEMENTS}

This work has been supported by the INFM Research Advanced Project on Bose-Einstein Condensation. 


\section{REFERENCES}

1. M.H. Anderson et al., Science 269, 189 (1995); K.B. Davis et al., Phys. Rev. Lett. 75, 3969 (1995); C.C. Bradley et al., Phys. Rev. Lett. 75, 1687 (1995).

2. T.C. Killian et al., Phys. Rev. Lett. 81, 3807 (1998); D.G. Fried et al., Phys. Rev. Lett. 81, 3811 (1998); T.J. Greytak et al., submitted to Physica B.

3. S. Giorgini, L.P. Pitaevskii, and S. Stringari, Phys. Rev. A 54, 4633 (1996); Phys. Rev. Lett. 78, 3987 (1997); J. Low Temp. Phys. 109, 309 (1997).

4. A. Griffin, Phys. Rev. B 53, 9341 (1996).

5. E.P. Gross, Nuovo Cimento 20, 454 (1961); J. Math. Phys. 4, 195 (1963); L.P. Pitaevskii, Zh. Eksp. Teor. Fiz. 40, 646 (1961) [Sov. Phys. JETP 13, 451 (1961)].

6. F. Dalfovo, S. Giorgini, L.P. Pitaevskii, and S. Stringari, Rev. Mod. Phys. 71, 463 (1999).

7. W. Krauth, Phys. Rev. Lett. 77, 3695 (1996); M. Holzmann, W. Krauth, and M. Naraschewski, Phys. Rev. A 59, 2956 (1999).

8. V. Bagnato, D.E. Pritchard and D. Kleppner, Phys. Rev. A 35, 4354 (1987); P.W.H. Pinkse et al., Phys. Rev. Lett. 78, 990 (1997).

9. T.W. Hijmans, Yu. Kagan, G.V. Shlyapnikov and J.T.M. Walraven, Phys. Rev. B 48, 12886 (1993).

10. R. Cotè and V. Kharchenko, Phys. Rev. Lett. 83, 2100 (1999).

11. A. Smerzi, S. Fantoni, S. Giovanazzi, and S.R. Shenoy, Phys. Rev. Lett. 79, 4950 (1997); S. Raghavan, A. Smerzi, S. Fantoni, and S.R. Shenoy, Phys. Rev. A 59, 620 (1999). 
12. L. Salasnich, A. Parola, and L. Reatto, Phys. Rev. A 60, 4171 (1999).

13. M.R. Andrews et al., Science 275, 637 (1997).

14. I. Zapata, F. Sols and A.J. Leggett, Phys. Rev. A 57, R28 (1998). 


\begin{tabular}{|cccccc|}
\hline \hline$T(\mu \mathrm{K})$ & $N_{0} / N$ & $E / N_{0}$ & $\mu$ & $\Gamma$ & $\tau_{1 / 2}$ \\
\hline 44.0 & 0.21 & 4.64 & 6.59 & 2.53 & 0.37 \\
44.5 & 0.18 & 4.39 & 6.25 & 2.08 & 0.38 \\
45.0 & 0.16 & 4.13 & 5.89 & 1.65 & 0.41 \\
45.5 & 0.13 & 3.82 & 5.46 & 1.27 & 0.44 \\
46.0 & 0.10 & 3.45 & 4.95 & 0.88 & 0.49 \\
46.5 & 0.07 & 3.04 & 4.37 & 0.56 & 0.56 \\
47.0 & 0.04 & 2.52 & 3.64 & 0.29 & 0.69 \\
47.5 & 0.02 & 1.89 & 2.75 & 0.10 & 0.93 \\
\hline \hline
\end{tabular}

TAB. 1. Condensate properties, for $N=2.2 \cdot 10^{10}$ interacting hydrogen atoms in the Ioffe-Pritchard trap, at various temperatures: condensed fraction $N_{0} / N$, energy per particle of the condensate $E / N_{0}$ and chemical potential $\mu$ in units of $10^{3} \hbar \omega_{z}$ (where $\left.10^{3} \hbar \omega_{z} / k_{B}=0.49 \mu \mathrm{K}\right)$, two-body decay rate $\Gamma$ in units of $10^{10} \mathrm{~s}^{-1}$ and decay time $\tau_{1 / 2}$ in seconds.

\begin{tabular}{|c|ccccccc|}
\hline \hline$N$ & $U_{0}$ & $E_{\text {int }}$ & $\Delta E^{0}$ & $\Lambda$ & $z_{c}$ & $z_{c} N$ & $\tau$ \\
\hline 10000 & 8 & 1.371 & 0.222 & 24.69 & 0.39 & 3900 & 0.09 \\
10000 & 10 & 1.383 & 0.164 & 33.64 & 0.34 & 3400 & 0.10 \\
10000 & 12 & 1.397 & 0.126 & 44.38 & 0.30 & 3000 & 0.12 \\
\hline 50000 & 16 & 4.345 & 0.095 & 183.03 & 0.15 & 7500 & 0.08 \\
50000 & 18 & 4.361 & 0.072 & 242.95 & 0.13 & 6500 & 0.09 \\
50000 & 20 & 4.378 & 0.055 & 317.80 & 0.11 & 5500 & 0.10 \\
\hline \hline
\end{tabular}

TAB. 2. Parameters of the MQT for $N=10^{4}$ and $N=5 \cdot 10^{4}$ condensed hydrogen atoms. Ioffe-Pritchard external potential and different values of the double-well potential barrier $U_{0}$. The laser-beam radius is $\sigma=5 \mu \mathrm{m}$. The energies are in units of $\hbar \omega_{z}$ and the oscillation period $\tau$ in seconds. 


\section{FIGURE CAPTIONS}

Fig. 1. BEC transition temperature $T_{c}$ versus number $N$ of hydrogen atoms. Comparison between harmonic and Ioffe-Pritchard trapping potential.

Fig. 2. Condensate density profile $n_{0}$ and thermal density profile $n_{T}$, for a cloud of $N=2.2 \cdot 10^{10}$ interacting hydrogen atoms in the Ioffe-Pritchard potential. Densities are in units of $a_{z}^{3}$. Notice the very different abscissae scales.

Fig. 3. Observables of the Bose condensate as a function of temperature $T$. $N=2.2 \cdot 10^{10}$ interacting hydrogen atoms in the Ioffe-Pritchard trap.

Fig. 4. Condensate fraction $N_{0} / N$ as a function of temperature $T$. $N$ interacting hydrogen atoms in the Ioffe-Pritchard trap. 


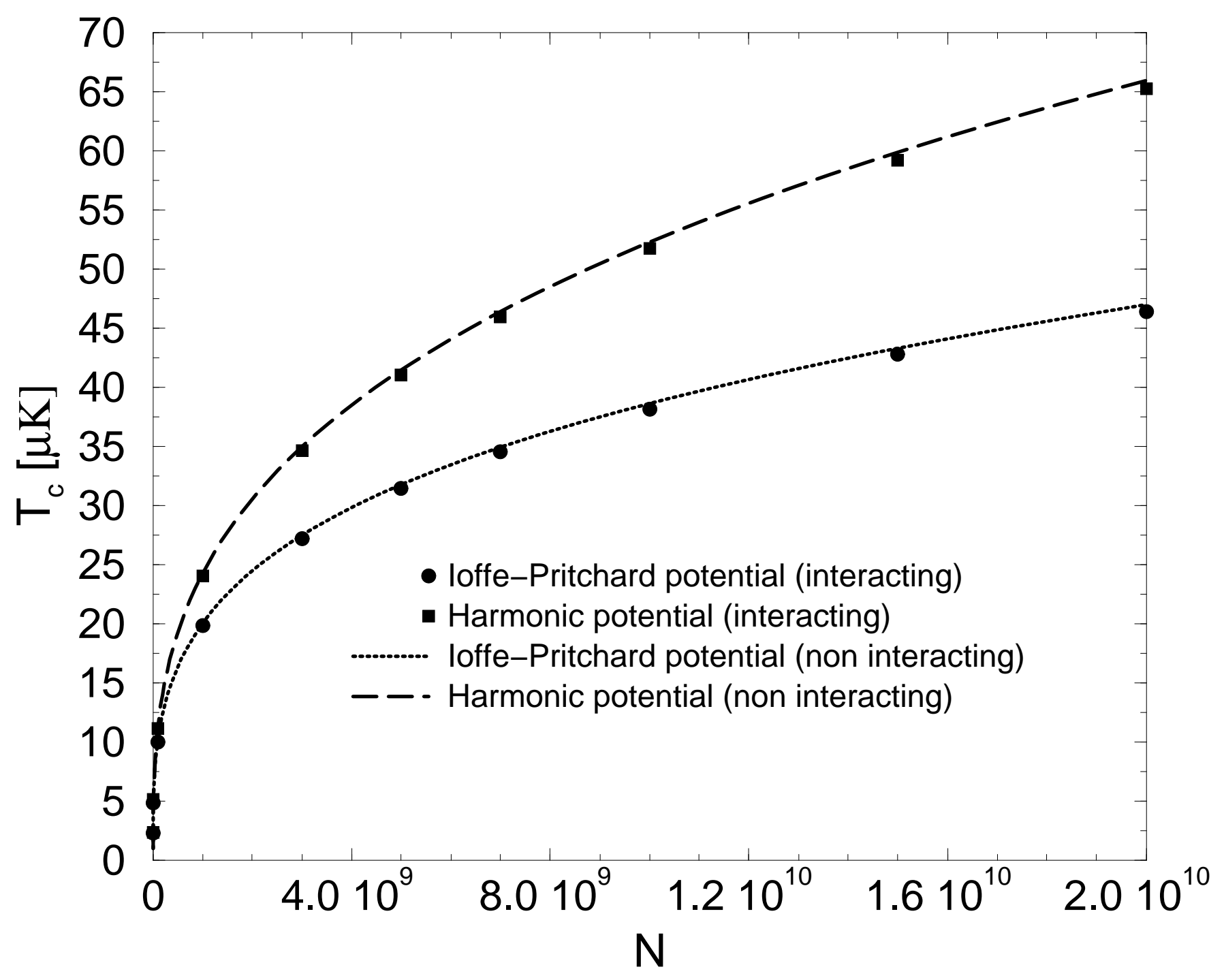



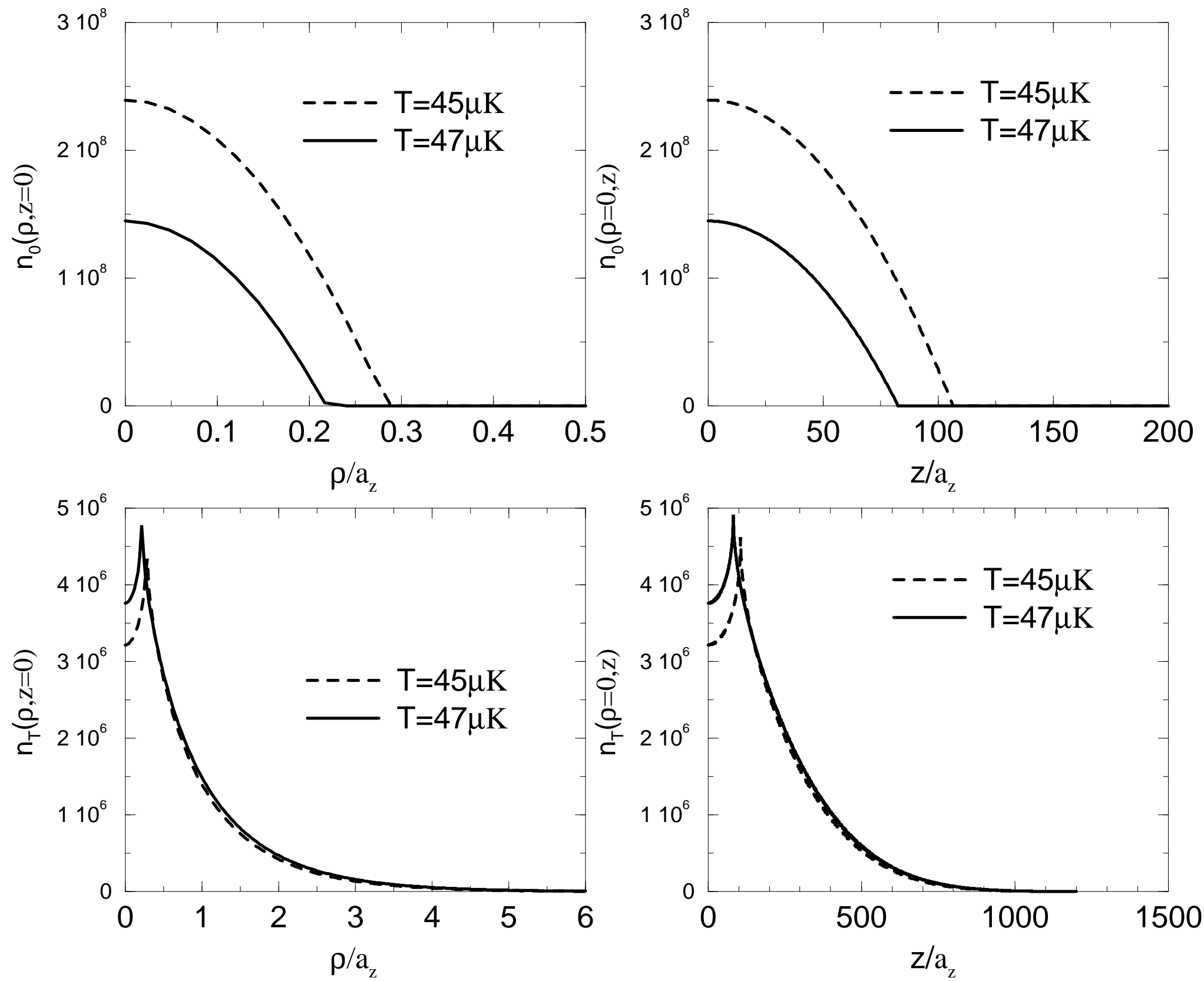


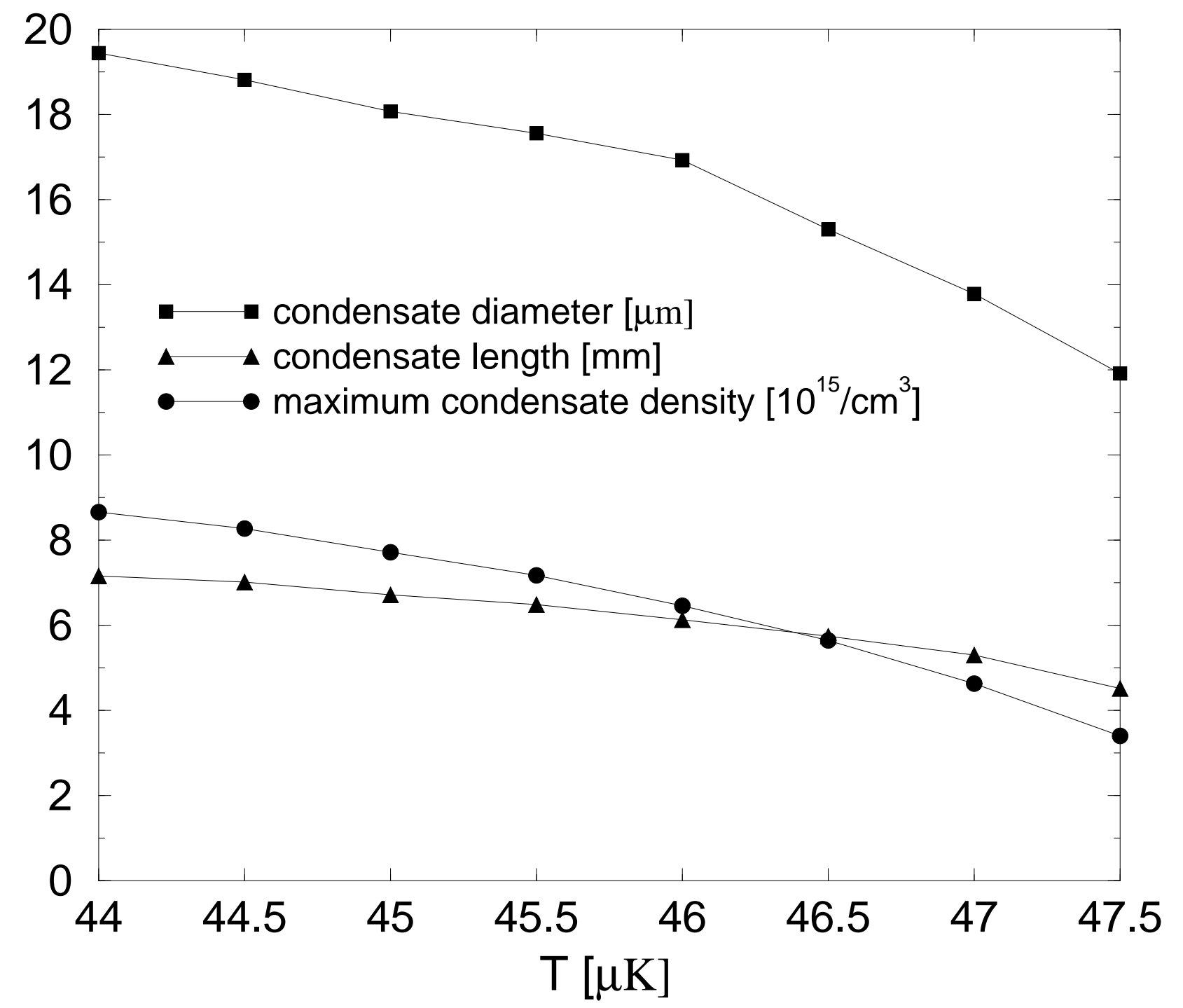




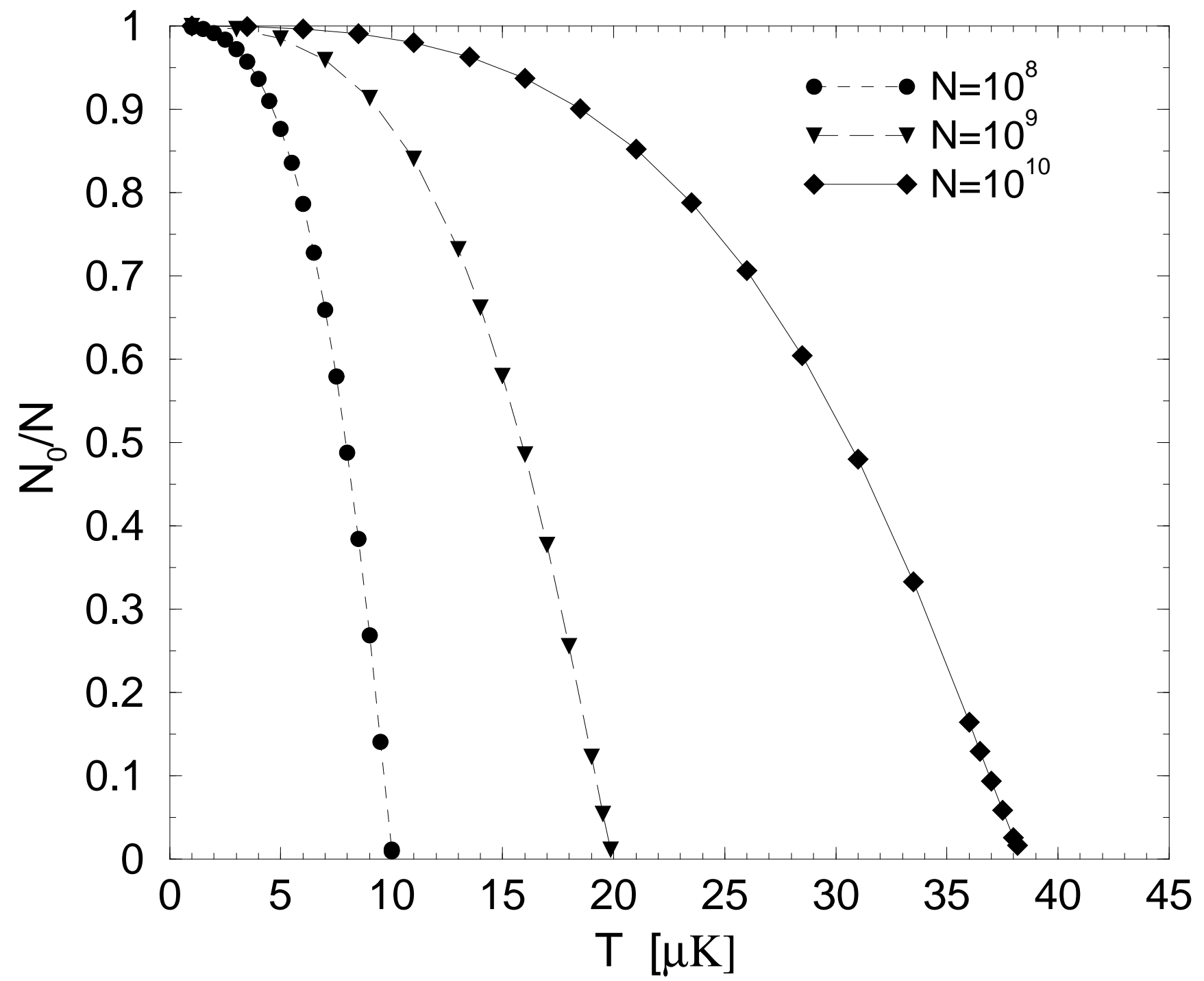

